\title{
Prenatal diagnosis of Roberts syndrome in a Chinese family based on ultrasound findings and whole exome sequencing: a case report
}

\author{
LiFen Zhu ${ }^{1,3}$, DingYa Cao ${ }^{4}$, Min Chen ${ }^{4}$, Huimin Zhang ${ }^{1,3}$, XiaoFang Sun ${ }^{1,3^{*}}$ and WeiQiang Liu $^{2^{*}}$ (1)
}

\begin{abstract}
Background: Roberts syndrome (RBS) is a rare autosomal recessive disorder caused by variations in the ESCO2 gene; however, prenatal diagnosis of RBS has never been reported in Chinese families. Additionally, fetal-specific phenotypic characteristics associated with ESCO2 variants have not been reported.

Case presentation: A fetus in a healthy, nonconsanguineous Chinese family with multiple serious congenital malformations was diagnosed prenatally. Two consecutive fetuses in this family presented with tetraphocomelia, growth restriction, cleft lip and palate bilaterally, and other abnormalities. The main phenotypic characteristics of this case were strongly suspected to be associated with RBS. Finally, whole exome sequence analysis revealed the insertion of a homozygous base pair in exon 6 of the ESCO2 gene (NM_001017420.3, c.1111 insA, NP_001017420.1, p.Thr371fs). Both of the couples were heterozygous carriers for this variant.
\end{abstract}

Conclusion: We are the first to report a prenatal case of RBS diagnosed in a Chinese family. Here, we have confirmed that the rare variant is a definite pathogenic variant, and we provide detailed phenotypic characteristics for the prenatal diagnosis of RBS due to this causative variant.

Keywords: Prenatal diagnosis, Roberts syndrome, Ultrasound, Whole exome sequencing, ESCO2

\section{Background}

Roberts syndrome (RBS, OMIM 268300) is a rare autosomal recessive disorder caused by variants in the establishment of the cohesion 1 homolog 2 (ESCO2) gene [1]. The main characteristics of RBS are prenatal and postnatal growth retardation, tetraphocomelia and craniofacial abnormalities (ranging from mild to severe). Hand

\footnotetext{
*Correspondence: xiaofangsun@gzhmu.edu.cn; liuwq06@126.com ${ }^{1}$ Department of Obstetrics and Gynecology, Key Laboratory for Major Obstetric Diseases of Guangdong Province, The Third Affiliated Hospital of Guangzhou Medical University, Guangzhou 510150, Guangdong, China

${ }^{2}$ Central Laboratory, Longgang District Maternity \& Child Healthcare Hospital of Shenzhen City, Shenzhen 518172, China

Full list of author information is available at the end of the article
}

abnormalities, ear abnormalities, and cardiac or kidney abnormalities may occasionally occur in affected individuals. The most serious RBS cases result in spontaneous abortion, stillbirth or death within 1 month [2]. However, some mildly affected patients can survive into adulthood with varying degrees of intellectual disability [3].

The prevalence of RBS is unclear. To date, approximately 150 cases of RBS have been reported in the literature [3]. Although the incidence of RBS is not affected by race, a Chinese case of RBS diagnosed prenatally has not yet been reported in the literature. Here, we present a case of RBS diagnosed prenatally with a severe phenotype in a Chinese family. The clinical diagnosis is based 
on fetal anomalies detected by ultrasound followed by a molecular diagnosis by whole exome sequencing.

\section{Case presentation}

A 29-year-old pregnant woman (G2P0) and her husband were referred to our fetal medicine department for genetic counseling. On the 22nd week of her first pregnancy, the female fetus had multiple serious congenital anomalies, including limb malformation, bilateral cleft lip and palate, and severe growth restriction. They opted for termination of pregnancy, and no other survey was conducted for the aborted fetus. The healthy, nonconsanguineous couple of Chinese origin denied any other family history of congenital diseases and had no history of exposure to medication during pregnancy.

During her current pregnancy, the woman did not have regular physical examinations. At 25 weeks of gestation, ultrasound examinations performed at the local hospital again showed multiple similar congenital malformations of the fetus. At the same time, a detailed ultrasonic examination conducted in our hospital showed a singleton male fetus with severe growth restriction, with a biparietal diameter (BPD) of $55.02 \mathrm{~mm}(-2.4 \mathrm{SD}$, the 0.8 percentile), occipito-frontal diameter (OFD) of $67.6 \mathrm{~mm}$ $(-4.0 \mathrm{SD}$, the 0.03 percentile), head circumference $(\mathrm{HC})$ of $195.4 \mathrm{~mm}(-4.0 \mathrm{SD}$, the 0.8 percentile), abdominal circumference (AC) of $155.23 \mathrm{~mm}(-3.9 \mathrm{SD}$, the 0.05 percentile) and estimated fetal weight (EFW) of $378 \mathrm{~g}(-1.7$ SD, the 4.457 percentile) (Fig. 1).

The fetus had flexion contractions in the elbow, knee and ankle on both sides, as well as with equinovarus talipes. The extremities were significantly shortened bilaterally in the humerus and femur. The length of the right and left humerus was $29.3 \mathrm{~mm}(-5.2 \mathrm{SD})$ and $31.3 \mathrm{~mm}$ $(-4.5 \mathrm{SD})$, while the length of the right and left femurs was $33.3 \mathrm{~mm}(-4.4 \mathrm{SD})$ and $33.9 \mathrm{~mm}(-4.1 \mathrm{SD})$, respectively. The bilateral radius, ulna and fibula were apparently not presented (Fig. 2).

Ultrasound examination of fetal hands revealed the absence of the right thumb and the presence of an appendicular thumb on the left. A bilateral cleft lip and cleft palate were also identified (Fig. 3). No internal abnormalities or polyhydramnios were observed, and the genitourinary system was normal.

The couple opted for pregnancy termination and decided to proceed with genetic testing to determine the causative genetic factors. Postmortem examination confirmed the ultrasound findings mentioned above.

For genetic assessment, $10 \mathrm{ml}$ of fetal amniotic fluid and $5 \mathrm{ml}$ of parents' peripheral blood were collected for fetal-only chromosomal microarray analysis (Thermo Fisher, Affymatrix Cytoscan $750 \mathrm{~K}$ ) and trio-whole exome sequencing (Mingma Technologies Co. Ltd) to detect copy number variations and gene variants, respectively. No pathogenic or likely pathogenic copy number variants were identified in the fetus. A trio-whole exome sequencing analysis revealed a homozygous frameshift insertion of a homozygous base pair in exon 6 of the ESCO2 gene (NM_001017420.3, c. 1111insA, NP_001017420.1, p. Thr371fs), and both couples were heterozygous carriers for this variant. Sanger sequencing was performed and confirmed this variant (Fig. 4).

\section{Discussion and conclusions}

RBS, also known as Roberts-SC phocomelia syndrome or ESCO2 spectrum disorder, is a rare autosomal recessive disorder caused by variants of the $E S C O 2$ gene [4]. Patients with RBS have a variety of signs, ranging from serious malformation to milder forms $[2,5,6]$. The pathogenesis of RBS is caused by a variant in the ESCO2 gene
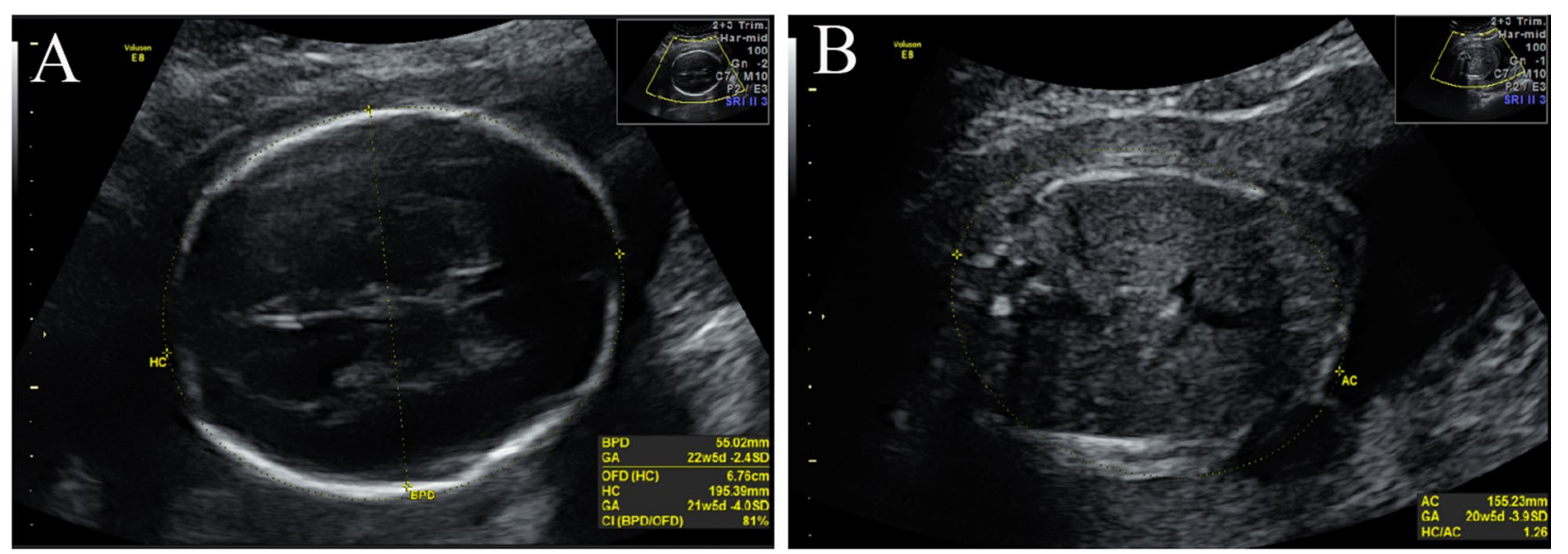

Fig. 1 Fetal ultrasound at 25 weeks of gestation indicates severe growth restriction. BPD, HC (A) and AC (B) measure below the $3 r d$ percentile 

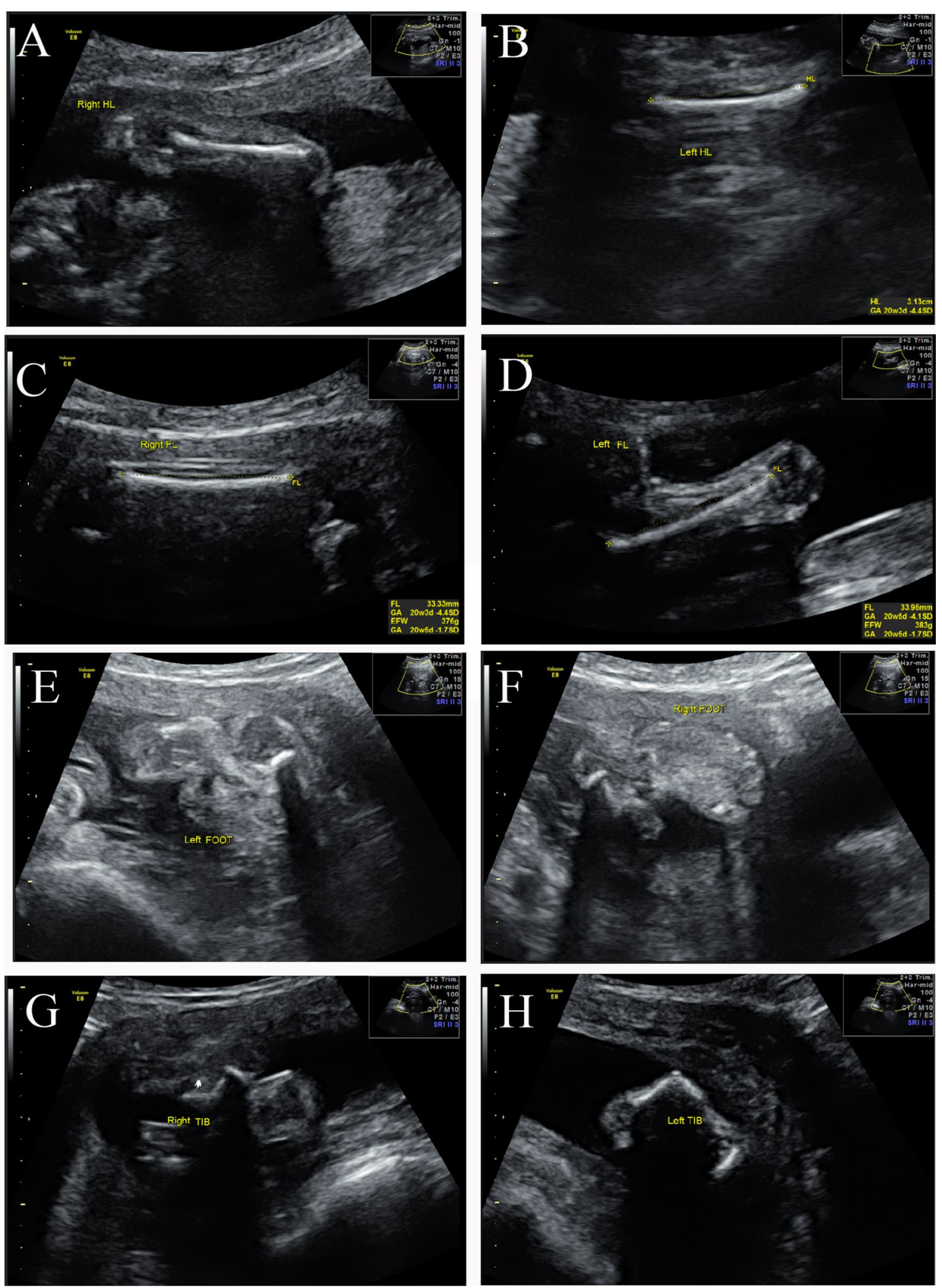

Fig. 2 The extremities were significantly reduced bilaterally in the humerus $(\mathbf{A}, \mathbf{B})$ and femur $(\mathbf{C}, \mathbf{D})$. The fetus had flexural contractions in the elbow, knee and ankle on both sides, as well as equinovarus talipes $(\mathbf{E}-\mathbf{H})$ 

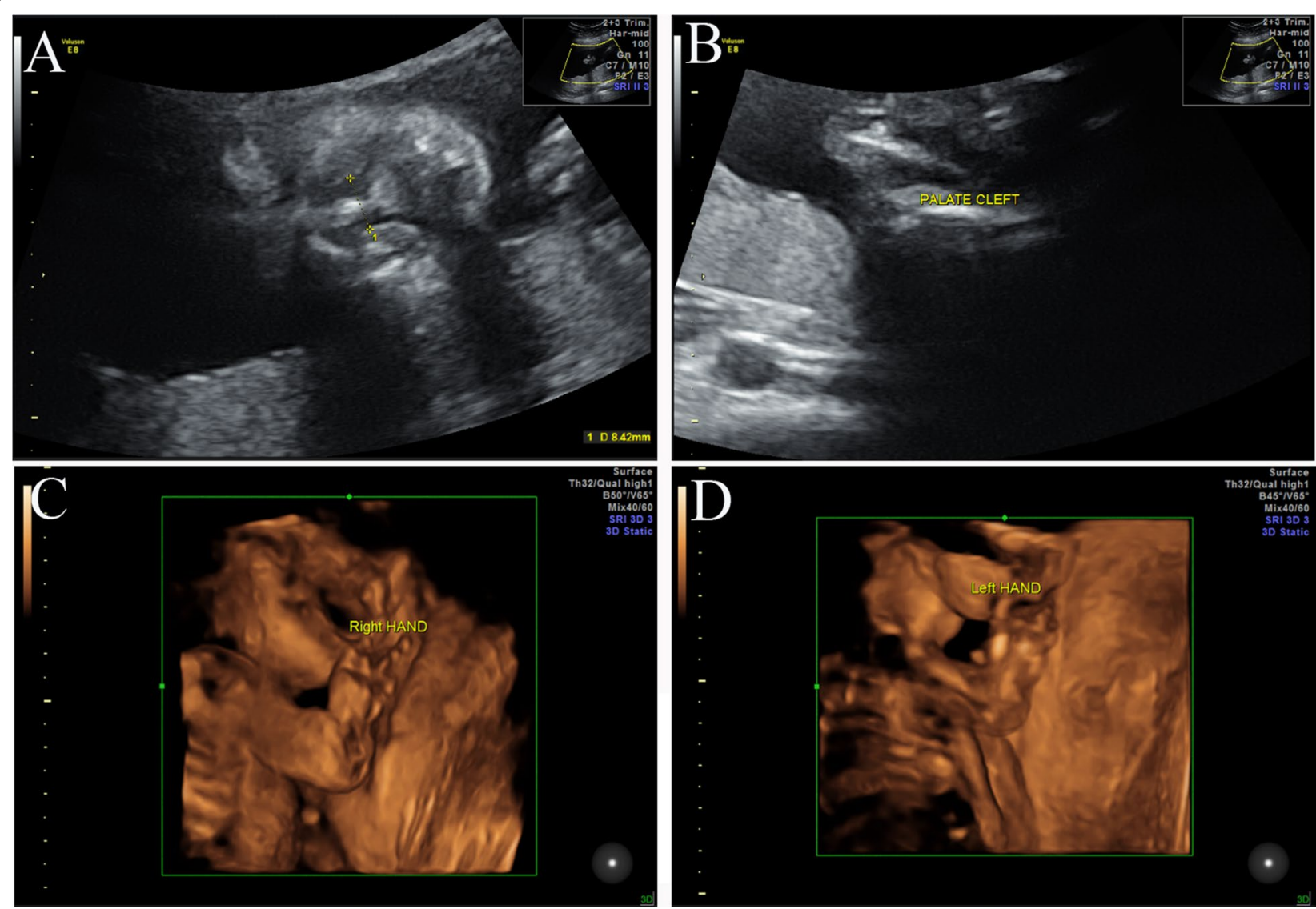

Fig. 3 Ultrasound examinations reveal a bilateral cleft lip and cleft palate in the fetus $(\mathbf{A}, \mathbf{B})$, the absence of the right thumb and the presence of an appendicular thumb on the left (C, D)

located at $8 \mathrm{p} 21.1$, which encodes a protein with the function of acetyl-transferase in establishing sister chromatid cohesion during $\mathrm{S}$ phase and mitosis [5]. In RBS patients, ESCO2 gene defects lead to the loss of acetyltransferase activity, sister chromatid polymerization failure and centromeric separation, and they result in very slow or even interrupted cell division and abnormal gene expression patterns [7].

For prenatal diagnosis of RBS, there must be careful differentiation between cases where the conditions are clinically similar. For example, limb anomalies and growth retardation can be observed in RBS, Cornelia de Lange syndrome, CHARGE syndrome, Tetra-melia syndrome 1 and thrombocytopenia-absent radius syndrome. Therefore, identification of the causal genetic factor plays a critical role in the clinical diagnosis or future preimplantation genetic testing of these diseases. In this case, a fetus in a healthy, nonconsanguineous Chinese family with multiple serious congenital malformations was diagnosed prenatally. Two consecutive fetuses in this family had tetraphocomelia, growth restriction, bilateral cleft lip and palate and other anomalies. Most of the clinical parameters observed are below the 3rd percentile. The key phenotypic features of our case are strongly suspected to be associated with RBS.

Although approximately 150 cases of RBS have been reported, the exact prevalence of RBS is unknown. To date, there are approximately 40 pathogenic or likely pathogenic ESCO2 variants listed in the ClinVar database (https://www.ncbi.nlm.nih.gov/clinvar) or in the literature $[8,9]$, and most of them are frameshift, nonsense or splice-site variants. Regarding our case, the c.1111dupA variant in exon 6 of the ESCO2 gene is not present in population databases (such as the ExAC and gnomAD databases). Although this variant has been reported three times in individuals with RBS, for which it has been classified as a pathogenic or likely pathogenic variant (https://www.ncbi.nlm.nih.gov/clinvar/variation/21232/), the fetal-specific phenotypic characteristics correlated with this variant have not been reported.

We reviewed the relevant literature and databases, and unfortunately, the prevalence of the disease and this variant in the Chinese population is not known. Although the couple denied consanguinity, we cannot rule out the 

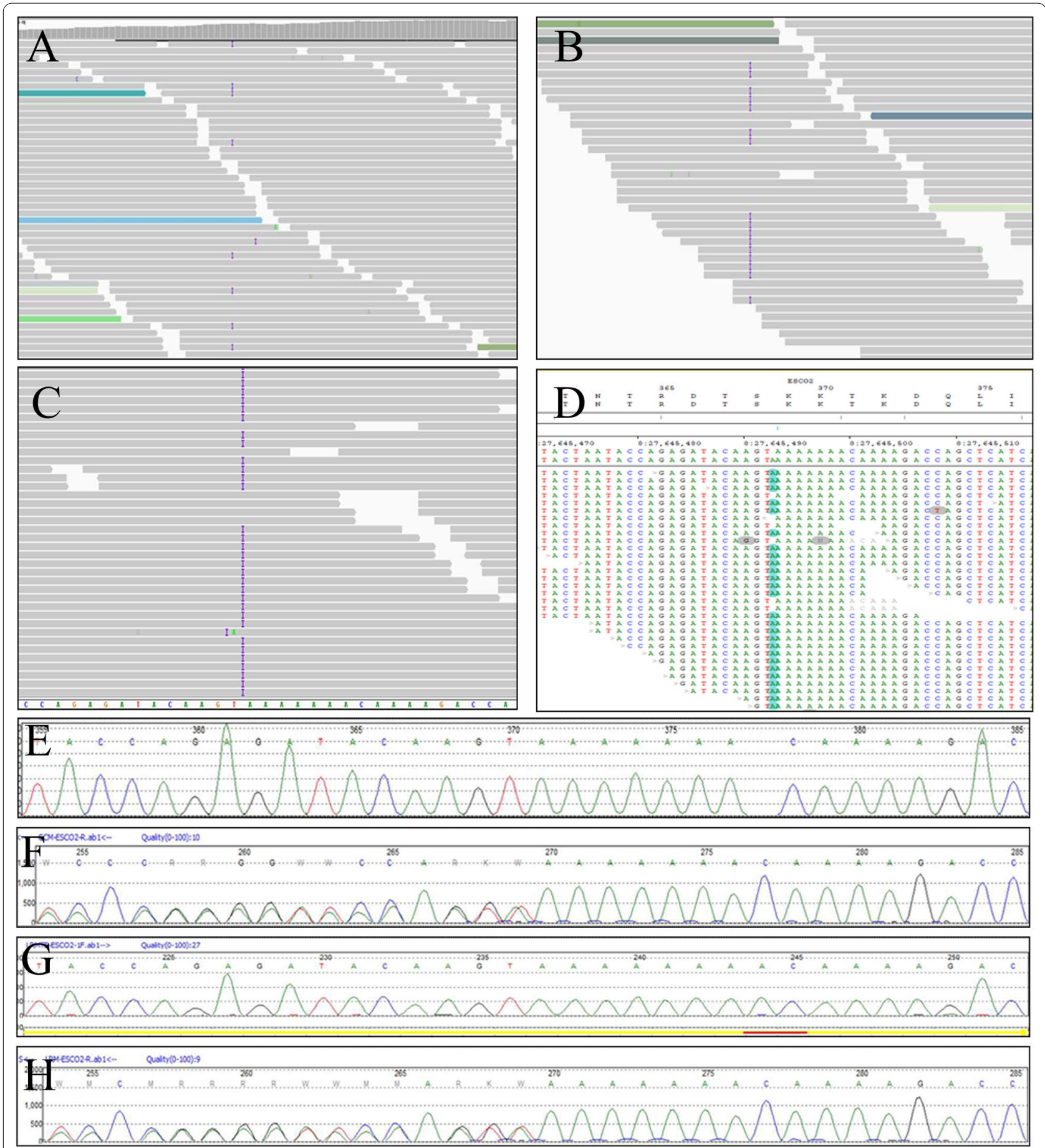

Fig. 4 Whole exome sequencing identified a heterozygosity frameshift insertion of 1 base pair involving exon 6 of the ESCO2 gene (NM_001017420.3, c.1111insA, NP_001017420.1, p.Thr371fs) in the couple (A, B) and a homozygous mutation of this allele site (C, D). Sanger sequencing confirmed the mutation. Both members of the couple were heterozygous for the mutation $(\mathbf{F}, \mathbf{H})$, and the fetus had a homozygous mutation (G). E Represents a reference sequence of the ESCO2 gene

possibility of a common ancestry between them, given that both members of the couple are carriers of the same very rare variant.
According to the gene variant interpretation guidelines [10] and the similar classical RBS phenotype observed in the consecutive two fetuses, we classified 
this variant as a definitive pathogenic variant based on the following criteria: PVS1-VS (pathogenic criterion is weighted as very strong), PP4 (patient's phenotype or family history is highly specific for a disease with a single genetic etiology), and PM2-supporting (absent from controls or population database if recessive in Exome Sequencing Project, 1000 Genomes Project, or Exome Aggregation Consortium).

Although the c.1111dupA variant has already been described once previously [4], the clinical information, particularly the phenotype present during pregnancy, is not fully provided. Based on detailed prenatal ultrasound results and molecular tests, we demonstrated that the c.1111dupA variant in the ESCO2 gene could cause a severe and classical phenotype of RBS.

Genetic testing of carrier status for the parents was indicated to calculate the risk of having another child with RBS in a future pregnancy. As the genetic analysis revealed that both parents are carriers of the c.1111dupA variant of the ESCO2 gene, the parents were informed that they have a $25 \%$ risk of having another affected child and were discussing options regarding a future pregnancy. In addition, fetal cells can be obtained by amniocentesis extraction of amniotic fluid, and cytogenetic analysis of fetal DNA, which is the gold standard for the diagnosis of RBS during pregnancy, can confirm the diagnosis [11].

In conclusion, we report, for the first time, a case of prenatally diagnosed RBS in a Chinese family. We have confirmed that the rare variant is a definite pathogenic variant and provided detailed phenotypic characteristics for the prenatal diagnosis of RBS due to this causative variant. Studies on the pathogenesis of such recurrent congenital malformations in whole exome sequencing provide the basis for genetic counseling to avoid the risk of recurrence and complications in future pregnancies.

\section{Abbreviations}

RBS: Roberts syndrome; BPD: Biparietal diameter; SD: Standard deviation; OFD: Occipito-frontal diameter; HC: Head circumference; AC: Abdominal circumference; EFW: Estimated fetal weight.

\section{Acknowledgements}

We thank the patient and her family for providing medical records and allowing us to report this case.

\section{Authors' contributions}

LFZ was responsible for the original draft preparation and editing. DYC; MC and HMZ managed the patient and obtained the patient's history; DYC and MC collected images; DYC and HMZ collected the blood samples and performed the DNA extraction experiment; LFZ, XFS, and WQL participated in data interpretation; XFS and WQL were responsible for conceptualization, manuscript editing, and reviewing the manuscript. All authors read and approved the final manuscript.

\section{Funding}

This work and publication costs were financially supported by Natural Science Foundation of Guangdong Province (2019A1515011302) and National Natural Science Foundation of China (32070582). The design of the study and writing of the manuscript were supported by both grants. Sample collection and experimental procedure (high-throughput exome sequencing), as well as data analysis and interpretation concerning Variable Site of the patient were supported by the Grant 2019A1515011302. The funding bodies played no role in the design of the study and collection, analysis, and interpretation of data and in writing the manuscript.

\section{Availability of data and materials}

All data generated or analyzed in this study are included in the published article. whole exome sequencing data for fetuses and parents of Variable Sites are available in the NCBI SRA under the accession numbers PRJNA788695 (https:// www.ncbi.nlm.nih.gov/Traces/study/?acc=PRJNA788695).

\section{Declarations}

Ethics approval and consent to participate

Written informed consent was obtained from the patient and her husband. The study was approved by the Ethics Committee of the Third Affiliated Hospital of Guangzhou Medical University (No: 2021043).

\section{Consent for publication}

Written consent for publication was obtained from the patient and her husband for publication of this study and its copy is available for the journal. These materials include characteristic phenotypic images, personal information, genetic testing results and other related data used in this report.

\section{Competing interests}

The authors report no competing interests.

\section{Author details}

${ }^{1}$ Department of Obstetrics and Gynecology, Key Laboratory for Major Obstetric Diseases of Guangdong Province, The Third Affiliated Hospital of Guangzhou Medical University, Guangzhou 510150, Guangdong, China. ${ }^{2}$ Central Laboratory, Longgang District Maternity \& Child Healthcare Hospital of Shenzhen City, Shenzhen 518172, China. ${ }^{3}$ Key Laboratory of Reproduction and Genetics of Guangdong Higher Education Institutes, Guangzhou 510150, China. ${ }^{4}$ Department of Obstetrics and Gynecology, Department of Fetal Medicine and Prenatal Diagnosis, Key Laboratory for Major Obstetric Diseases of Guangdong Province, The Third Affiliated Hospital of Guangzhou Medical University, Guangzhou 510150, China.

Received: 4 July 2021 Accepted: 7 January 2022

Published online: 29 January 2022

\section{References}

1. Vega H, Gordillo M, Jabs EW. ESCO2 spectrum disorder. In: Adam MP, Ardinger HH, Pagon RA, Wallace SE, Bean LJH, Mirzaa G, Amemiya A, editors. GeneReviews((R)). Seattle: University of Washington; 1993.

2. da Costa Almeida CB, Welter AT, Abech GD, Brandao GR, Flores JAM, Schule B, Francke U, Fiegenbaum M, Zen PRG, Rosa RFM. Report of the phenotype of a patient with Roberts syndrome and a rare ESCO2 variant. J Pediatr Genet. 2020;9(1):58-62.

3. Zhou J, Yang X, Jin X, Jia Z, Lu H, Qi Z. Long-term survival after corrective surgeries in two patients with severe deformities due to Roberts syndrome: a case report and review of the literature. Exp Ther Med. 2018;15(2):1702-11.

4. Vega H, Waisfisz Q, Gordillo M, Sakai N, Yanagihara I, Yamada M, van Gosliga D, Kayserili H, Xu C, Ozono K, et al. Roberts syndrome is caused by mutations in ESCO2, a human homolog of yeast ECO1 that is essential for the establishment of sister chromatid cohesion. Nat Genet. 2005;37(5):468-70.

5. Goh ES, Li C, Horsburgh S, Kasai Y, Kolomietz E, Morel CF. The Roberts syndrome/SC phocomelia spectrum - a case report of an adult with review of the literature. Am J Med Genet Part A. 2010;152a(2):472-8. 
6. Schneeberger PE, Nayak SS, Fuchs S, Kutsche K, Girisha KM. Roberts syndrome in an Indian patient with humeroradial synostosis, congenital elbow contractures and a novel homozygous splice variant in ESCO2. Am J Med Genet A. 2020;182(11):2793-6.

7. Nasmyth $\mathrm{K}$, Haering $\mathrm{CH}$. Cohesin: its roles and mechanisms. Annu Rev Genet. 2009;43:525-58.

8. Afif HH, Abdel-Salam GM, Eid MM, Tosson AM, Shousha WG, Abdel Azeem AA, Farag MK, Mehrez MI, Gaber KR. Expanding the mutation and clinical spectrum of Roberts syndrome. Congenit Anom. 2016;56(4):154-62.

9. Vega H, Trainer AH, Gordillo M, Crosier M, Kayserili H, Skovby F, Uzielli ML, Schnur RE, Manouvrier S, Blair E, et al. Phenotypic variability in 49 cases of ESCO2 mutations, including novel missense and codon deletion in the acetyltransferase domain, correlates with ESCO2 expression and establishes the clinical criteria for Roberts syndrome. J Med Genet. 2010;47(1):30-7.

10. Richards S, Aziz N, Bale S, Bick D, Das S, Gastier-Foster J, Grody WW, Hegde $\mathrm{M}$, Lyon E, Spector E, et al. Standards and guidelines for the interpretation of sequence variants: a joint consensus recommendation of the American College of Medical Genetics and Genomics and the Association for Molecular Pathology. Genet Med Off J Am Coll Med Genet. 2015;17(5):405-24.

11. Percival SM, Thomas HR, Amsterdam A, Carroll AJ, Lees JA, Yost HJ, Parant JM. Variations in dysfunction of sister chromatid cohesion in esco2 mutant zebrafish reflect the phenotypic diversity of Roberts syndrome. Dis Model Mech. 2015;8(8):941-55.

\section{Publisher's Note}

Springer Nature remains neutral with regard to jurisdictional claims in published maps and institutional affiliations.

- fast, convenient online submission

- thorough peer review by experienced researchers in your field

- rapid publication on acceptance

- support for research data, including large and complex data types

- gold Open Access which fosters wider collaboration and increased citations

- maximum visibility for your research: over $100 \mathrm{M}$ website views per year

At BMC, research is always in progress.

Learn more biomedcentral.com/submissions 